\title{
The use of the Planetarium in Navigation Instruction
}

\author{
from Captain F. D. Wilkinson \\ (South African Nautical College, General Botha)
}

1. INTRODUCTION. The planetarium consists of an optical projector centrally situated under a dome. 1 The viewers sit around the projector instrument in chairs designed to give a comfortable view over the whole interior. The instrument used by the writer is the Spitz A2, manufactured by Spitz Laboratories, Inc., Delaware, U.S.A. 2 Seating is provided for 40 persons under a $20-\mathrm{ft}$. dome in the particular installation at the South African Nautical College General Botha, Gordon's Bay, Cape.

Instruction is given to the college students and visiting naval and air-force officers and the facilities are available to schools and other organized bodies who make regular use of them. This note deals with the use of the instrument in teaching astro-navigation.

2. Advantages. The planetarium is a teaching aid which makes a tremendous impact on the viewer. It offers great interest to a student, as the artificial sky is both impressive and fascinating. The celestial sphere is demonstrated from any latitude, and the aid is excellent for showing the diurnal movements of the celestial bodies at the poles (turntable or circumpolar motion), at the equator (tilting motion) and at intermediate latitudes (twisting motion). A demonstration gyroscope mounted on a meridian ring ${ }^{3}$ emulates the movements of the planetarium sky and the connection provides a valuable adjunct to gyro-compass instruction.

The planetarium enables the teacher to demonstrate the geometry of the celestial sphere and is helpful in explaining elements of spherical geometry. One can interpret quite remarkably well the principles of astronomy apart from those of navigation. 4

3. Description of THe Instrument. The sky contains the stars visible to the naked eye. Stars of first and second magnitude are mostly provided with individual projectors given their correct colour and brightness. Five bright planets, Moon and Sun are projected independently and the Moon projector has phasereproducing attachments. An arrow-shaped indicator is used as a pointer and held in the hand.

The teacher is able to draw numerous lines in the sky, the most important being the celestial meridian, the coordinate system and spherical triangles. Diurnal circles, vertical circles, and in fact any great or small circle may be shown. An additional projector made locally from an ordinary torch fitted with a suitable mask is used with great success to plot a position circle or the path of a circumpolar star.

A sextant projector held in the hand measures angles on the sphere. A map of the world viewed geocentrically, i.e. with the viewer's eye at the centre of the Earth may be projected on to the dome and a D.R. position anywhere is selected as convenient. The position of the elevated pole is indicated by a spot of white light. The zenith-spot projector of the instrument was adapted in the college workshops to enable it to depress sufficiently to indicate the pole at various 
altitudes. The zenith point may be also indicated with the meridan projector. The moon phase unit (Earth-lit Moon) was also adapted for use as the Mean Sun in demonstrating the subject of Time.

The coordinate projector projects the ecliptic, equinoctial, First Point of Aries, First Point of Libra, short arcs of hour circles which are numbered both in right ascension and sidereal hour angle, and the parallels of declination to $30^{\circ}$ north and south. The equinoctial is graduated for more accurate readings. It is pointed out to students that the coordinates thus drawn in the sky are in effect the Nautical or Air Almanac in graphical form. It is extremely helpful to a student for him to see the Almanac as a picture of lines instead of a mass of figures. The positions of the heavenly bodies may be read off directly in the sky.

4. Lectures. Most of the nautical astronomy section of the Ministry of Transport syllabus, the illustrations contained in any navigation text-book or manual drawn on the plane of the rational horizon, and those suitable for air navigation may be reproduced easily in the planetarium sky. Thirteen separate lectures are delivered to students training for the Merchant Service whilst similar lectures are given to naval and air-force students. In every lecture opportunity is taken to practice star identification. Experience shows the instrument is without equal in this respect.

As an example of what can be achieved the following is a brief description of an actual demonstration.

On a table alongside the control panel are displayed the tools of the trade necessary to observe and plot an astronomical position; the book of the Almanac, a chronometer, sextant and globe of the Earth. Each one is simulated by visible means in the sky.

The sky is moved into suitable aspect at twilight and a star selected. Identification of the particular star is determined and the sky stopped rotating. It is explained that the age-old custom of calling 'stop' is tantamount to stopping the sky.moving, giving a particular instant in time; and hence a specific shape to the triangle for the observation. This provides a quick understanding of the fundamental necessity for the information presented by the Almanac and chronometer, for without these the shape could not be determined and sight reduction would be impossible. The struggle to provide the correct time to the seaman is seen in its true perspective.

The sextant projector is used to find the angle of altitude and it is shown that this does nothing more than measure the length of a line; the arc of the vertical circle from the horizon to the star. The line is drawn in the sky. It is also demonstrated that swinging the sextant gently from side to side is simply to determine the precise position of the foot of the vertical circle on the horizon. The line is continued $90^{\circ}$ up to the zenith spot (D.R. position) and the 'altitude line' deleted, leaving a line drawn from the zenith to the star: the zenith distance. This forms one side of the triangle.

The position of the elevated pole having been previously indicated by a spot of white light in Antarctica and the equinoctial's position in the sky having been aligned to the Earth's equator on the geocentric map, the student now has no difficulty in accepting their respective positions among the stars. Furthermore, the relationship between the equatorial system and the horizon system of determining the star's position becomes plain.

A second line (arc of a celestial meridian) is drawn connecting the star to the equinoctial. The length of this is readily seen as the declination and its value may 
be read off the grid of lines thrown into the sky. This line is extended to the pole and the 'declination line' expunged.

The arc of the local meridian from the horizon to the pole spot is drawn and this too extended up to the zenith. The 'altitude of the pole line' is deleted leaving the celestial triangle glowing overhead in lines of light against a backcloth of stars.

The demonstration covers a detailed description of the angles and sides. The actual angles formed may be measured in degrees along the horizon or equinoctial by projecting the appropriate great circles forming the triangle on to these parts. The sextant projector may be held horizontally (or obliquely) to measure angles and thus simulate the mariners' compass in measuring the azimuth.

A setting of the sky as viewed from the pole may be used to show the relationship between G.H.A. Aries, S.H.A. Star, longitude and L.H.A. The shape of the triangle may be varied by moving the star towards or away from the meridian until on the meridian the triangle obviously vanishes.

Resuming the discussion the teacher shows how the two sides and included angle for Marc St. Hilaire, or the three sides for Longitude by Chronometer are used for solving the triangle. The intercept is derived by comparing visually the actual length of the line found by calculation with the true zenith distance line measured by the sextant. It is plotted towards or away as the case may be.

The final step in the problem is solved by projecting the map of the world on to the sky. The star's position on the Earth (G.P.) is immediately apparent. With the torch projector the position circle is drawn. It is of interest here to note that the size of the triangle and position circle is usually enormous, a fact seen at a glance by their appearance on the map. A ship in the South Atlantic (the zenith spot indicating her position) has a triangle stretching away down to the South Pole and perhaps right over to the South Indian Ocean. The position circle is obviously as large at normal altitudes. Thus the student may be made readily aware of the 'secret' of a sight reduction in that to alter the position of the zenith spot or pole spot slightly does not alter the material size of that enormous triangle. In other words, the D.R. position may be in the proximity of the true position without affecting the concept of the computation. This is so obvious in the life-size planetarium yet seldom satisfactorily explained on a class-room blackboard.

Detailed discussion of observational errors 5 is usually confined to more advanced students in planetarium lectures, although some errors are readily illustrated to anyone.

Finally the short arc of the position circle at right angles to the radius line shows the navigator's plot on his local chart. A similar azimuth line and position line crossing the first determines the position.

5. Time. The subject as taught in a class-room bristles with difficulties, and most elementary students and even more advanced ones have little more than a superficial concept of time. The planetarium enables the teacher rapidly to demonstrate the apparent motion of the sky because a day passes in about four minutes. The passage of the First Point of Aries, the Mean Sun and the Apparent Sun across the local meridian or the Greenwich Meridian can be most adequately and forcefully demonstrated. The 'hand of the master clock of the sky' is shown sweeping across the celestial sphere, the angle it makes with the 'reference line' (the meridian) being the time. ${ }^{4}$ The Equation of Time is visible as the angle contained between the 'hands' of the Mean and Apparent Suns. The Dynamical 
Mean Sun ${ }^{6}$ is also made visible. The clock stars are shown in transit, and the viewer appears to be sitting at the viewing end of a transit circle. At the same instant Aries can be seen and its distance from the meridian is self-evident. As the months are marked along the ecliptic the celestial time-keepers are shown in the sky in their correct positions and their relative movements throughout the year are easily seen. Ancillary equipment such as a sundial and a wooden clockface with a painted reference line and the time indicator (the hour hand) suffice to make clear the connection between the sky-clock and a watch.

6. Miscellaneous. The movements of the Moon are complex and difficult for a student to grasp from book descriptions. It is possible to demonstrate satisfactorily the difference between the Synodic and sidereal periods of revolution and show the eastward progress of the Sun and Moon against the backdrop of stars; the Moon chasing the slow-moving Sun. As the stars and planets can be made visible during the time the Sun is above the horizon the movements of the Sun and Moon among the stars manifest themselves without the necessity to deduce them. 6 A proper understanding of their movements is found to help considerably in such matters as the study of tides and eclipses.

A 'sputnik' projector gives a realistic satellite and this, although seemingly of more academic than professional interest to the navigator, permits discussion of the possibility of navigation by satellite fixes. 7

7. The room in which the planetarium is housed has been fitted out as a navigation museum. The walls carry magnificent photographs of a variety of sky objects including a mosaic photograph of the Moon 3 feet in diameter. Navigation instruments and models from an early age are on display. An exhibition of the creation of a mercator chart from the original sounding boards through to the printed chart is provided through the good offices of the Hydrographer of the South African Navy. Various other exhibitions such as Glenn's orbit of the Earth are displayed as topical features. Lectures are augmented by films. The stimulus the planetarium provides creates a centre of activity and learning and serves to promote good will for the college within the community. 8

8. Conclusion. No amount of descriptive material can replace the actual lectures given under the planetarium sky or do justice to the instrument. Where a city has a permanent installation it may possibly be used to further an understanding of astro-navigation provided a lecturer with a suitable professional background is available. As a student begins to become aware of mathematical concepts the quantitative aspects of his observations in the planetarium take on a different emphasis. Repeated visits are essential and each lecture will be an experience, especially to an impressionable young student.

\section{REFERENCES}

1 King, H. C. (1960). 'The Development of the Planetarium.' The Journal of the British Astronomical Association, 70, 1 .

2 The Use of the Planetarium in the Teaching of Earth and Space Sciences, (1960). Spitz Laboratories, Inc., U.S.A.

3 The Demonstration Gyroscope and Meridian Ring, Sperry Gyroscope Co., Ltd., Brentford.

4 Baker, R. H., Astronomy, D. van Nostrand Co.

5 H.O. Pub. No. 9. Bowditch, U.S. Navy Hydrographic Office.

6 Admiralty Manual of Navigation, Vol. III. B.R. 45/3.

7 Scott, W. A. (1961). This Journal, 14, 87.

8 Williams, Herbert N., (1960). The Planetarium in Modern Education, American School Boord Journal, U.S.A. 